\title{
Eine Spitalrechnung, die zu denken gibt
}

\section{Hanswerner Iff}

Dr. med., Mitglied FMH

Wir Ärzte und Ärztinnen sind uns gewohnt, nach bestem Wissen unsere Patienten zu betreuen, und erwarten zu Recht, dass wir für dieses Tun auch vernünftig entlohnt werden. Manchmal erhalten wir auch Spitalrechnungen, weil wir selbst der Behandlung bedürfen. Meistens sind diese nicht nur für Patienten schwer verständlich, sondern auch für Arzt und Ärztin selbst. Im Artikel wird versucht, eine Rechnung zu verstehen und wichtige Gesichtspunkte zu diskutieren.

\section{Die Spitalrechnung}

Die Rechnung wurde für einen etwas mehr als 25 Stunden dauernden, also stationären, Spitalaufenthalt erstellt. Als therapeutische Leistungen kam eine elektrophysiologische Untersuchung mit ablativen Massnamen bei einer störenden Herzrhythmusstörung zur Verrechnung. Dafür wurden Herzkatheterinterventionen von den inguinalen Gefässen durchgeführt. Die Intervention brauchte grosse Geschicklichkeit und war zweifellos sowohl materiell aufwendig als auch personalintensiv. Die in Kopie wiedergegebene Rechnung wurde für einen Spitalaufenthalt des Autors erstellt. Sie ist im Text, partiell anonymisiert, integriert.

Ich habe, wie jeder Einwohner der Schweiz, die obligatorische Grundversicherung (nach KVG) und dazu eine Zusatzversicherung (nach VVG). Diese beiden Versicherungen standen somit zur Bezahlung des erwähnten Spitalaufenthaltes zur Verfügung. Die Spitalrechnung erhielt ich von der Krankenkasse auf mein Verlangen zugeschickt.

Die in Kopie wiedergegebene Rechnung wurde für einen Spitalaufenthalt des Autors erstellt. Sie ist im Text, partiell anonymisiert, integriert.

In dieser sind Einzelposten und pauschalisierte Leistungen in bunter Reihenfolge aufgelistet. Beim ersten Blick ist es schwer ersichtlich, wie der Rechnungssteller die erbrachten Leistungen zwischen der Grundund der Zusatzversicherung in einen Gesamtzusammenhang bringt. Mit den Umrandungen wird etwas Klarheit geschaffen. Die orange Umrandung trennt die
Rechnungsbeträge der Fallpauschale von denjenigen der Zusatzversicherung, die blau umrandet sind. Für die Fallpauschale ergibt sich ein Total (abzüglich SwissDRG) von CHF 9527.15, das, etwas verwirrlich, noch aufgeteilt wird. Die gesamten Kosten des Spitalaufenthaltes ergeben sich schliesslich aus den übrigen Zwischentotalen (Tages- und Pflegetaxen, Infrastruktur, Honorare, Medikamente, Material und Spezialmaterial/Katheter). Der Gesamtbetrag dieser Leistungen (dick blau umrandet) beträgt CHF 17612.00. Erst in der

In der Spitalrechnung sind Einzelposten und pauschalisierte Leistungen in bunter Reihenfolge aufgelistet.

abschliessenden Kostenübernahme (unten, orange umrandet) wird ersichtlich, warum die Fallpauschale in der Detailrechnung aufgeteilt ist. Es handelt sich um die gesetzlich vorgegebene Aufteilung der Fallpauschalen zwischen Kanton (55\%) und obligatorischer Grundversicherung (45\%) bei stationären Behandlungen. Diese Beträge (Krankenkasse GV und Gesundheitsund Fürsorgedirektion KT) werden dann in der «Kostenübernahme» von den gesamten Leistungen abgezogen, so dass für die Zusatzversicherung (Krankenkasse $\mathrm{ZV}$, dick rot umrandet) schliesslich der Betrag von CHF 8084.90 anfällt.

\section{Weitere Fakten zur Rechnung}

Rückfragen bei der Krankenkasse ergaben, dass die Spitalrechnung der Norm entspreche und aus ihrer Sicht nicht beanstandet werden könne. Ein Bezug auf gesetzliche Vorgaben wurde nicht gemacht. Bei der 


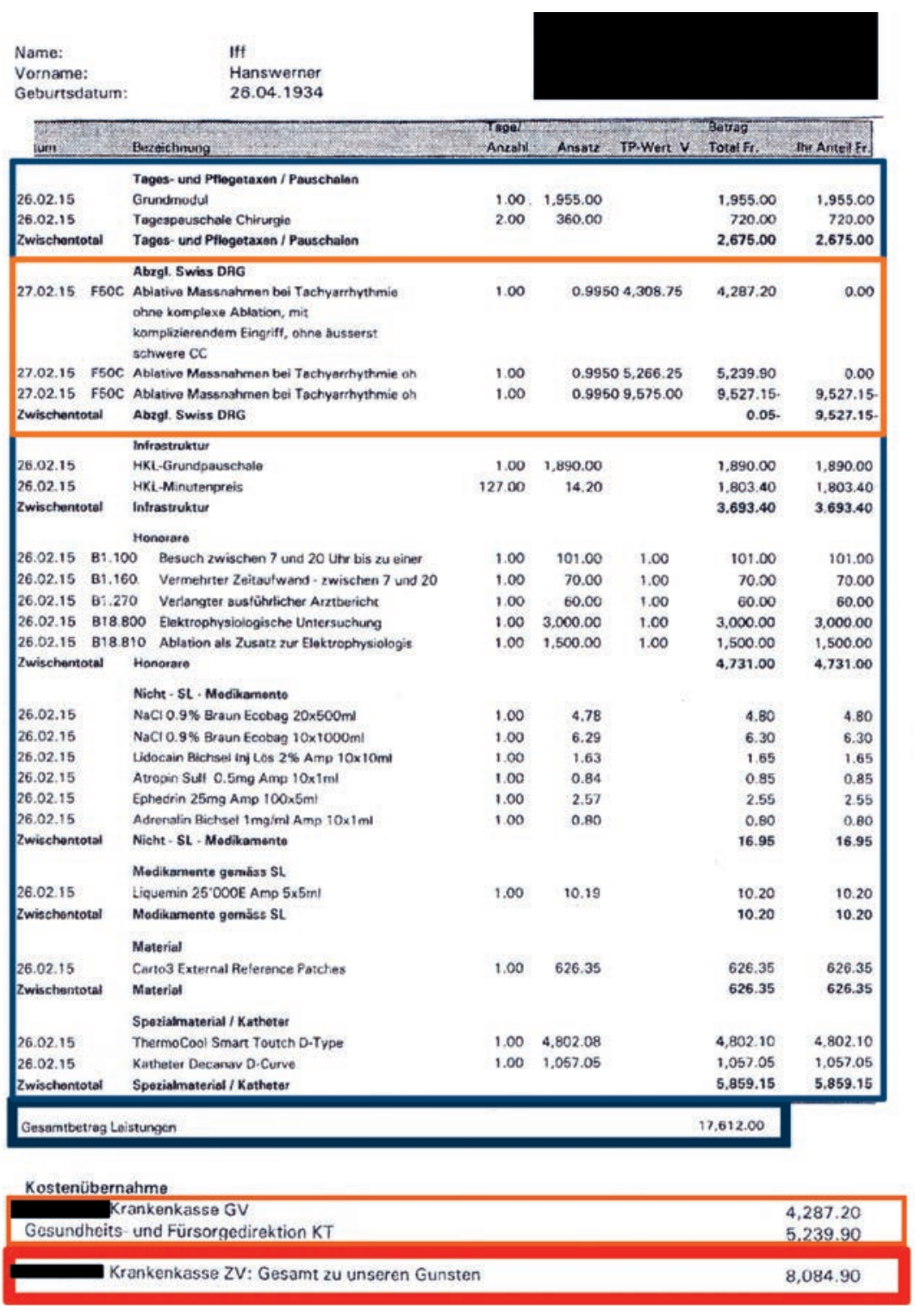

Kopie der Spitalrechnung: Legende siehe Text.
Rücksprache mit der Spitalverwaltung wurde mir gesagt, dass ich aufgrund meiner Zusatzversicherung eine frei wählbare und exzellente ärztliche, apparative und pflegerische Betreuung gehabt hätte, in einem

Rückfragen bei der Krankenkasse ergaben, dass die Spitalrechnung der Norm entspreche und aus ihrer Sicht nicht beanstandet werden könne.

Zweierzimmer und nicht in einem Mehrbettzimmer gewesen sei und von einer guten Küche profitierte. Das mag ja alles stimmen. Doch bleibt hier ein Unbehagen zurück.

Mit der Grundversicherung hat jeder Patient und jede Patientin, die stationär hospitalisiert werden, das An- recht auf eine gute Spitalversorgung, deren Preis nach bestem Wissen von der SwissDRG festgelegt wird. Die schweizweite, tarfwirksame Einführung von SwissDRG ist 2012 erfolgt. Das KVG (1994, Stand am 1. Januar 2018) definiert deren Aufgaben zusammengefasst folgendermassen: Die SwissDRG AG ist zuständig für die Erarbeitung und Weiterentwicklung sowie die Anpassung des Fallpauschalen-Systems zur Abgeltung der stationären Spitalleistungen. Sie bringt ein sachgerechtes und gesetzeskonformes Abgeltungssystem für eine faire Finanzierung von stationären Spitalleistungen ein und ist eine gemeinsame Institution der Leistungserbringer, der Versicherer und der Kantone im schweizerischen Gesundheitssystem.

Unter dem Begriff der «Zusatzversicherung» (ZV), der in der Rechnung, zwar abgekürzt, aber doch mit klarem Hinweis, wie auch in der Police meiner Krankenkasse unter "Pflegezusatzversicherung», steht, verstehe ich Zusätze, die zu einem definierten Tarif eben zusätzlich in Rechnung gestellt werden. Eventuelle Gründe für eine andere Auslegung fehlen jedenfalls auf der Rechnung. Inwiefern dies, aufgrund des Versicherungsvertragsgesetzes (VVG, 1908 [!], Stand 1. Januar 2011, in parlamentarischer Beratung) rechtens ist, ist mir nicht bekannt. In den Kundeninformationen der Krankenversicherung (von 2007) steht, dass «der Versicherer die für die schweizerische Sozialversicherung gültigen Tarife sowie die üblich angewendeten Privattarife anerkennt».

Ich begreife und begrüsse die Tatsache, dass zusätzliche Spitalleistungen erbracht und auch versichert werden können. Das kann eine ärztliche und pflegerische Mehrleistung, die Wahl eines bestimmten Arztes, ein gastronomischer Vorteil, eine (noch) nicht anerkannte therapeutische Vorgehensweise oder eine teurere Form der medikamentösen Versorgung sein, auch andere definierte Spitalbehandlungen und Einzelzimmer erfassen, die ausserhalb der Grundleistungen sind, mehr Komfort bringen oder zu einer nicht obligaten Nachhospitalisation oder der Behandlung in einem anderen Kanton gehören. Solche Mehrleistungen und Vorteilsversorgungen sollten in einer Spitalrechnung aber einen durchschaubaren Bezug zur Grundversicherung haben. In vorliegender Rechnung wird in der Kostenübernahme einzig von Grundversicherung (GV) und Zusatzversicherung (ZV) gesprochen. Ein Bezug wird nicht erstellt.

\section{Wie soll sich die Grund- zur Zusatzversicherung verhalten?}

Die wichtigste Frage ist, ob die Zusatzversicherung eine eigenständige Versicherung ist, die erlaubt, prak- 
tisch unabhängig nach eigener Tarifierung Rechnung zu stellen. Von einem juristischen Standpunkt aus kann ich diese Frage nicht beantworten, doch vom ärztlich-medizinischen einige Gesichtspunkte einbringen.

Aus der Rechnung ist ersichtlich, dass das Verhältnis Zusatzversicherung (ZV) zu Grundversicherung (GV und KT) $45,1 \%$ zu 54,9\% beträgt. Das ergibt auf jeden Franken, der mir als Grundversichertem verrechnet wird, Zusatzleistungen von 84 Rappen. Diese Höhe der Zusatzleistungen überrascht. Ist es überhaupt möglich,

Aus der Rechnung ist ersichtlich, dass das Verhältnis Zusatzversicherung (ZV) zu Grundversicherung (GV und KT) 45,1\% zu 54,9\% beträgt.

innerhalb eines Tages solch kostenintensive zusätzliche Leistungen $\mathrm{zu}$ erbringen? Oder anders herum gefragt: Wie würde dann die Rechnung bei einem «nur» Grundversicherten aussehen? Erhält dieser Patient wirklich nur etwa die Hälfte «Leistung», wenn er nur grundversichert ist?

Die medizinisch-ärztliche Leistung und auch die pflegerische Betreuung sind zum Grossteil und zu Recht von Richtlinien gesteuert. Dazu werden die Leistungen des Arztes und der Ärztin durch entsprechende Examen der Fachgesellschaften, durch geordnete obligate Angaben zu Fortbildung und Weiterbildung (SIWF) und in Spitälern mit Ausbildungsverpflichtungen von akademischen Lehrern und Lehrerinnen im streng überwachten Assistenzverfahren kontrolliert. Dabei haben Arzt und Ärztin auf jeder Stufe den Ehrgeiz, beste Arbeit zu leisten. Wäre das nicht so, hätten sie den falschen Beruf gewählt. Die oberste Maxime des Arztberufes, auf welcher Stufe er auch ausgeführt wird, bleibt die gute Sorge für Patient und Patientin, und zwar für jeden Patienten und für jede Patientin in gleichem Masse. Mit diesen Vorgaben ist es nicht gegeben, dass grosse Zusatzleistungen, wie im vorliegenden Fall, bei kurzen Aufenthalten im ärztlichen, materiellen und pflegerischen Bereich erbracht werden können.

Zu Zeiten von Gotthelf, in Anne Bäbi Jowäger, 1844, war die Frage des ärztlichen Honorars noch einfach und ganz in den Händen vom Doktor und Patient. Gotthelf beschreibt ein Gespräch um eine Honorarforderung nach der geglückten Heilung einer alten, armen Frau mit deren Sohn. "Glebt müsset Ihr o ha», sagt dieser nach der Ablehnung eines bescheidenen Geldbetrages zum Doktor. «Habt nicht Kummer für mich, ich fordere dann bei einer reichen Frau desto mehr!», war die Antwort des engagierten Arztes. So sahen Zusatzleistung vor 150 Jahren aus, solidarisch und für die damaligen sozialen Verhältnisse auch gerecht.

Die Zeiten haben sich geändert. Heute sind nicht mehr nur zwei am Honorar beteiligt, sondern fast unübersichtlich viele. So ist bei der besprochenen Rechnung die Situation zweifelsohne komplizierter. Aber: Muss hingenommen werden, dass vom leistungserbringenden Spital bei einem zusatzversicherten Patienten die Kosten für seinen Aufenthalt im Vergleich zum «nur» Grundversicherten fast verdoppelt werden? Zudem werden diese Kosten buchhalterisch nicht als Zusätze deklariert. Woher ihre Beträge stammen, bleibt unklar und für den Patienten unkontrollierbar. Unweigerlich stellen sich hier wichtige Fragen: Deckt die Fallpauschale der Grundversicherung die Kosten bei einem definierten Eingriff im Spital ungenügend? Müsste diese erhöht werden? Oder bringt die Pflegezusatzversicherung die Möglichkeit einer übertrieben hohen Rechnung? Sollte diese konsequenter als Zusatz, was durch dieses Wort ja gegeben ist, an die Grundversicherung angepasst werden?

Was wir sicher nicht wollen, ist eine Krankenversorgung, in der nur Zusatzversicherte eine adäquate Behandlung erhalten! Oder soll der nur Grundversicherte mit aufbereitetem Occasionsmaterial, das eigentlich zum einmaligen Gebrauch bestimmt wäre, behandelt werden oder Schrittmacher und Gelenkprothesen von Verstorbenen implantiert erhalten?

\section{Fazit}

Zusatzversicherungen können zur Ergänzung der Grundversicherung sinnvoll sein. Werden sie aber als solche angewandt, sollten sie in Abhängigkeit zur Grundversicherung auch ausgewiesen werden. Der Zusammenhang zwischen Grundversicherung und den versicherungstechnischen Zusätzen muss klar ersichtlich sein. Nur so behält die Grundversicherung die von ihr erwarteten qualitativen Anforderungen. Jedenfalls sollte es nicht gestattet sein, mit einer von der Grundversicherung getrennten «Privat-Versicherung» eine unkontrollierbare Tarifierungen zu ermöglichen und diese dann als Zusatzversicherung zu deklarieren. Eine solche Auslegung der Zusatzversicherung gefährdet die medizinische Qualität, die von der Grundversicherung erwartet wird, und schadet dem Solidaritätsgedanken, der in dieser Versicherung steckt. 